\title{
Glutathione Enzymes and Liver Injury in Acute Dengue Viral Infection
}

\author{
Lal Chandrasena ${ }^{1 *}$, Arjuna De Silva ${ }^{2}$, Chandima De Mel ${ }^{1}$, Hemantha Peiris ${ }^{3}$, Visula Abesuriya ${ }^{1}$, \\ Sanjay De $\mathrm{Mel}^{4}$, Suranjith Seneviratne ${ }^{5}$, Samantha Bandara ${ }^{6}$
}

${ }^{1}$ Nawaloka Hospitals Research and Education Foundation, Nawaloka Hospitals PLC, Colombo, Sri Lanka

${ }^{2}$ Department of Medicine, Faculty of Medicine, University of Kelaniya, Kelaniya, Sri Lanka

${ }^{3}$ Department of Biochemistry, Faculty of Medical Sciences, University of Sri Jayewardenepura, Nugegoda, Sri Lanka

${ }^{4}$ Department of Haematology-Oncology, National University Cancer Institute, National University Health System Singapore, Singapore

${ }^{5}$ Institute of Immunity and Transplantation, Royal Free Hospital and University College London, London, UK

${ }^{6}$ Department of Medical Laboratory Sciences, Faculty of Allied Health Sciences, University of Sri Jayewardenepura, Nugegoda, Sri Lanka

Email: *dgmnh@sltnet.lk

How to cite this paper: Chandrasena, L., De Silva, A., De Mel, C., Peiris, H., Abesuriya, V., De Mel, S., Seneviratne, S. and Bandara, S. (2019) Glutathione Enzymes and Liver Injury in Acute Dengue Viral Infection. Journal of Biosciences and Medicines, 7, 61-71.

https://doi.org/10.4236/jbm.2019.710006

Received: September 7, 2019

Accepted: October 15, 2019

Published: October 18, 2019

Copyright $\odot 2019$ by author(s) and Scientific Research Publishing Inc. This work is licensed under the Creative Commons Attribution International License (CC BY 4.0).

http://creativecommons.org/licenses/by/4.0/

\begin{abstract}
Identification of redox markers may be of clinical significance in the management of dengue patients. This study is to identify the association between antioxidant enzymes, hematological parameters and liver transaminases in patients with acute dengue infection. Blood samples were taken from patients on the day of admission, day 05 and 07 from admission for analysis of glutathione peroxidase (GPX), glutathione reductase (GR), aspartate transaminase (AST), alanine transaminase (ALT) and hematological parameters. AST and ALT levels were significantly elevated $(\mathrm{p}<0.05)$ on day 05 in dengue patients. In contrast, GPX and GR showed significantly low levels on day 05 compared to on the day of admission and day 07. Although there was a decline in the trend of platelets towards day 05 , values were not significantly different. Dengue associated with liver injury appears to peak around day 05 when the GPX and GR enzymes levels in patients were the lowest suggesting that increased viral load in the acute phase of dengue infection has initiated an antioxidant imbalance. Thus, timely investigation of antioxidant enzymes (GR and GPX) and liver transaminases around day 05 of admission may be of value in the management of patients with dengue infection similar to as seen in platelet counts.
\end{abstract}

\section{Keywords}

Aspartate Transaminase, Alanine Transaminase, Dengue,

Glutathione Peroxidase, Glutathione Reductase 


\section{Introduction}

Dengue virus is a major mosquito-borne disease worldwide, with a thirty-fold increase in incidence during the past 50 years [1]. Dengue infections can be caused by any of the four closely related dengue viral serotypes [2] [3]. The initial, or primary, infection may cause symptoms from mild to severe. Subsequent infections with other sero types, called secondary dengue infection, may lead to more severe diseases [4], such as dengue hemorrhagic fever (DHF) or dengue shock syndrome (DSS). Varying degrees of liver involvement are reported during acute dengue infection and thought to be due to hypoxic damage due to impaired liver perfusion resulting from fluid leakage, oxidative stress or immune mediated injury [5].

The severity of dengue infection varies widely, suggesting immune pathological, genetic and viral type factors may play a role in disease severity [6] [7] [8] [9]. Host genetic factors, such as glucose 6-phosphate dehydrogenase deficiency, may also contribute to increased replication of dengue virus in monocytes, which effect abnormal cellular redox equilibrium. This regulates virus replication and virulence in cells [10] [11] [12]. Oxidative damage may affect all biochemical compounds of cells and causes the loss of fluidity and leads to the destruction of cell membrane because of structural deformity [13] [14]. Inactivation and removal of these ROS depend on relations involving a wide spectrum of antioxidative defense mechanisms. These ROS are neutralized by various mechanisms; GPx and GR have been reported to be a more sensitive antioxidant enzyme in dengue and other viral infections [15].

Glutathione (GSH), also known as $\gamma$-L-glutamyl-L-cysteinyl-glycine, is required in the maintenance and regulation of the thiol-redox status [16]. Under physiological conditions, the reduced form of GSH is the predominant form with its concentration from 10 to 100 fold higher than the oxidized form of GSH (GSSG). Under physiological conditions, the GSSG is produced by the catalyze of GSH peroxidase which is commonly known as Glutathione peroxidase (GPX). The production of the oxidized form of GSH (GSSG) requires a cysteine residue which at physiological $\mathrm{pH}$ is present as a thiolate form. Cysteine form of thiloate (N-acetyl-1-cysteine) will be used to replenish the glutathione concentration by the liver [17]. Under oxidative stress, these residues are prone to oxidation in sulfenic acid, which efficiently reacts with GSH leading to form GSSG which is catalytically reduced back to GSH by the NADPH dependent glutathione reductase (GR). Thus, the ratio between GSH to GSSG at 10:100 is an important indicator of the redox environment [18]. In this context, the depletion of GR and GPX levels appeared to be the primary mediators of cell damage which support our hypothesis that GR and GPX represent the most important in the maintenance and regulation of thiol-redox status due to differential redox species.

Alterations in the homeostasis of the GR and GPX group of antioxidant enzymes, have been implicated in the enterology and or progression of many human diseases e.g. cataract [19], cardio vascular disease [20] and dengue fever 
[21]. These studies have shown that reduced levels of GSH and GPX contribute to oxidative stress associated with many pathological statuses including dengue infection. Oxidants play a complex role in viral diseases by influences on host cell metabolism, viral replication and extending to desirable inactivating effects on viruses and less desired toxic effects on host tissue [22]. Assessment of liver function will be more vital in the case of pathogenesis of dengue viral infection. Thus, we decided to investigate the association of GR and GPX activity with respect to liver damage in acute dengue infection by using aspartate transaminase (AST) and alanine transaminase (ALT).

\section{Material and Method}

\subsection{Subjects}

A hospital-based single prospective cohort study was carried out on 48 patients (Male-29, Female-19), that Nawaloka Hospital, Colombo, Sri Lanka. All patients/guardians provided informed written consent for participation in the study.

\subsection{Ethical Clearance}

Ethical clearance for the study was obtained from the Ethics Review Committee, Nawaloka Hospitals, Colombo 02, Sri Lanka (Reference Number: NHREF/9/ $17 / 01)$

Study setting: Nawaloka Hospitals, Plc, Colombo 02, Sri Lanka.

Inclusion criteria: Age 12 years and above. Patients presenting on day 1 or 2 of fever positive for dengue antigen (NS1) as the test sensitivity of NS1 antigen test is high only day 1 and 2 .

Exclusion criteria: Chronic liver disease, pregnancy, steroid therapy and those who on more than the recommended dose were excluded.

\subsection{Collection of Blood Samples for Laboratory Investigations}

Venous blood was drawn from patients serologically confirmed positive by dengue NS1 rapid test (SD-bioline, Korea) on day of admission, day 05 of admission (at the lowest platelet count) and day 07 of admission for following laboratory analysis as this is correlated with the pattern of thrombocytopenia in dengue infection.

\section{1) Antioxidant enzymes (GPX and GR)}

Blood samples were collected into lithium heparin tubes and immediately stored in aliquot at $-4^{\circ} \mathrm{C}$ pending analysis for GPX and GR. Plasma GPX and GR antioxidants concentrations were determined by Randox commercial assay kit (Ransel test kit, Randox Laboratoris, UK) on Dimension clinical chemistry analyzer (Germany).

\section{2) Liver transaminases}

The serum sample was used to detect AST and ALT (Siemens, Germany) by the Dimension clinical chemistry analyzer. As in accordance with the manufactures 
procedure of assay kit, U/L was used.

\section{3) Hemaotological parameters}

The blood samples collected into EDTA tubes were used to assess the full blood count using Sysmex haematology analyzer (XS500, Japan).

\subsection{Statistical Analysis}

Analysis of data was performed using IBM SPSS statistics version 25. Since the probability-probability (PP) plot revealed the variables involved were not normally distributed, the Friedman test was used to compare the means between the on admission, day and day 07 of admission. Results were reported as median (IQR) and differences. A p-value $<0.05$ was considered significant.

\section{Result}

The study was carried out among 48 (20 males, 28 females, age range 12 - 69) patients who had dengue infection confirmed by positivity for NS1. The patients had fever for $\leq 3$ days at the time of enrolment. According to WHO 2009 Guidelines, 38 of patients were diagnosed as dengue fever (DF) and 10 were developed dengue hemorrhagic fever (DHF).

1) Patterns of changes in liver transaminase and antioxidants levels during acute phase ( 0 - 7 days)

The changes in liver transaminases (AST, ALT) and antioxidant enzymes (GPX and GR) were measured on the $3^{\text {rd }}, 5^{\text {th }}$ and $7^{\text {th }}$ days after onset of fever.

The results of liver transaminase pattern of changes and antioxidant levels in blood of dengue patients are shown in Table 1.

There was a significant increase in AST $(\mathrm{p}=0.001)$ and ALT $(\mathrm{p}=0.000)$ levels on day 5 of admission compare to day of admission (Figure 1(a) and Figure 1(b)). Gradually both AST and ALT declined on day 07 of admission while ALT had a significant reduction $(\mathrm{p}=0.013$ ) compared to the day 05 of admission. Both GSH (Figure 1(c), p = 0.01) and GPX (Figure $1(\mathrm{~d}), \mathrm{p}=0.03$ ) significantly declined in the day 05 of admission compared to day of admission. However, the GPX and GR levels increased in patients at the time of discharge compared to day 05 of admission.

\section{2) Pattern of changes in hemaotological parameters}

The changes in hematological parameters during the period are shown in $\mathrm{Ta}$ ble 1. The platelet count is accordance with the pattern of change observed in the antioxidant enzymes (Figure $1(\mathrm{e})$ ). The plalets were significantly reduced on the day 05 of admission and were increased on day 07 of admission. There were 04 patients who had platelet counts less than 50,000 on admission. Eight (08) patient's platelet counts were reduced to less than 50,000 in the day 05 of admission. Based on the WHO 2009 guidelines there were 08 patients who had evidence of fluid leakage by ultra sound scanning. Patients were, therefore, divided into two groups: positive fluid leakage $(n=10)$ and negative fluid leakage $(n=$ 38).

When considering antioxidants, the GPX level was significantly higher on the 
day of admission and day 05 of admission patients who had fluid leakage compared to other group. Similarly, high values of GR were observed in patients who had fluid leakage compared to others. The median percentage of platelet reduction on day 05 of admission was $26 \%(2.27 \%-85 \%)$ from the day of admission.

Table 1. Liver transaminases, antioxidant enzymesand Haemotogical parameters.

\begin{tabular}{ccccc}
\hline & $\begin{array}{c}\text { On admission Median } \\
(\min -\max )\end{array}$ & $\begin{array}{c}\text { Day 05 of admission } \\
\text { Median (min - max })\end{array}$ & $\begin{array}{c}\text { Day 07 of admission } \\
\text { Median (min - max })\end{array}$ & p-value \\
\hline ALT (U/L) & $63(18-279)$ & $105(25-644)^{\mathrm{a}}$ & $132(28-516)$ & $\mathrm{p}=0.000^{\mathrm{a}}$ \\
AST (U/L) & $63(19-265)$ & $102(25-622)^{\mathrm{b}}$ & $97(30-298)$ & $\mathrm{p}=0.000^{\mathrm{b}}$ \\
GR (U/L) & $4.8(12-226)$ & $4.6(15-219)^{\mathrm{c}}$ & $4.6(14-287)$ & $\mathrm{p}=0.002^{\mathrm{c}}$ \\
GPX (U/L) & $690(30-850)$ & $695(41-882)^{\mathrm{d}}$ & $675(45-840)$ & $\mathrm{p}=0.000^{\mathrm{d}}$ \\
Platelet (per $\mu \mathrm{l})$ & 150,000 & 90,000 & 110,000 & $\mathrm{p}=0.001^{\mathrm{e}}$ \\
Hametocrit (\%) & $40,000-246,000)$ & $(18,000-140,000)^{\mathrm{e}}$ & $(24,000-340,000)$ & \\
Haemoglobin & $13.3(10-16.5)$ & $13.9(10.5-16.9)$ & $13.5(10.8-16.8)$ & $\mathrm{p}=0.401$ \\
$(\mathrm{~g} / \mathrm{dL})$ & & $41.6(31.3-49.1)$ & $40.6(34-47.6)$ & $\mathrm{p}=0.560$ \\
WBC (per $\mu \mathrm{l})$ & $3025(1200-7100)$ & $3100(1300-11,750)$ & $4800(1600-17,000)$ & $\mathrm{p}=0.610$ \\
\hline
\end{tabular}

a,b,c,d,e-The values in day 05 of admission were significantly different $(\mathrm{p}<0.05)$ compared to on admission and day 07 of admission. 1 unit $(\mathrm{U})$ is the amount of enzyme that catalyzes the reaction of $1 \mu \mathrm{mol}$ of substrate per minute.

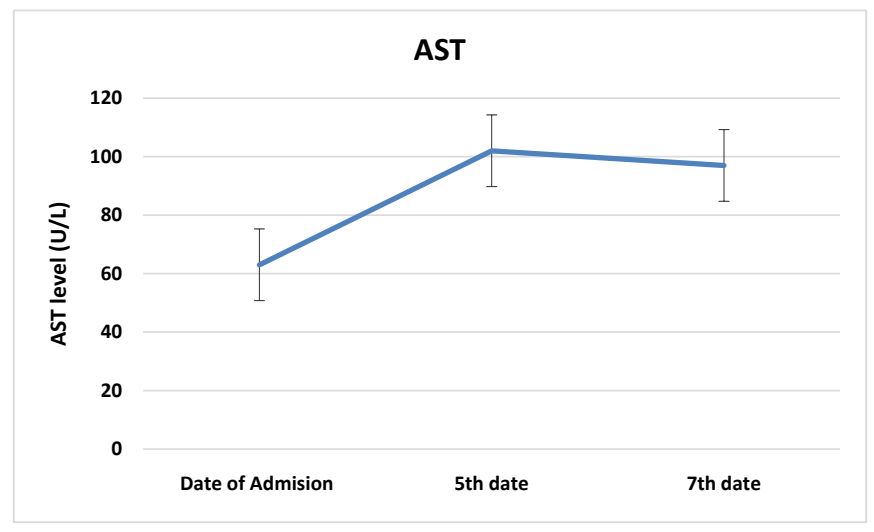

(a)

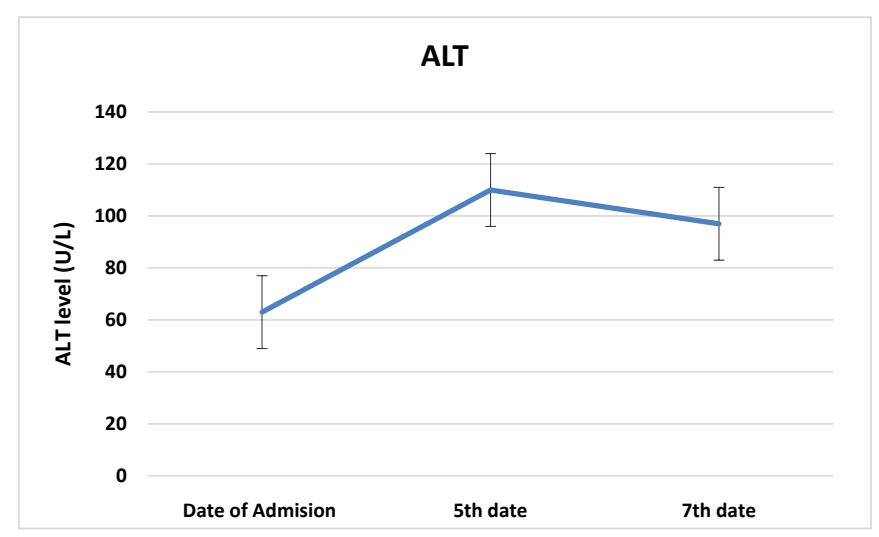

(b) 


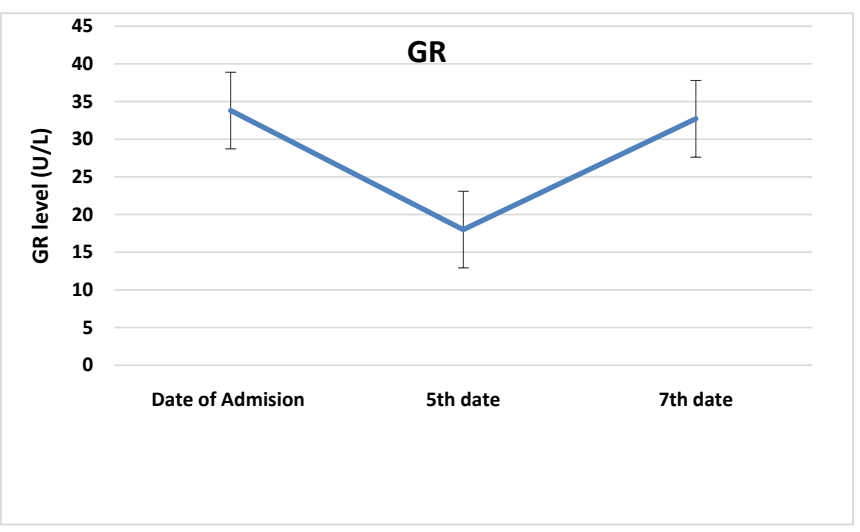

(c)

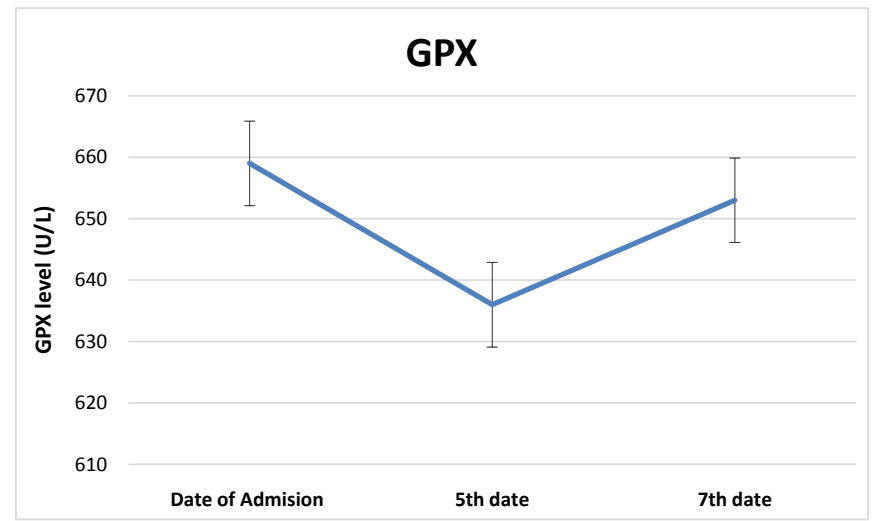

(d)

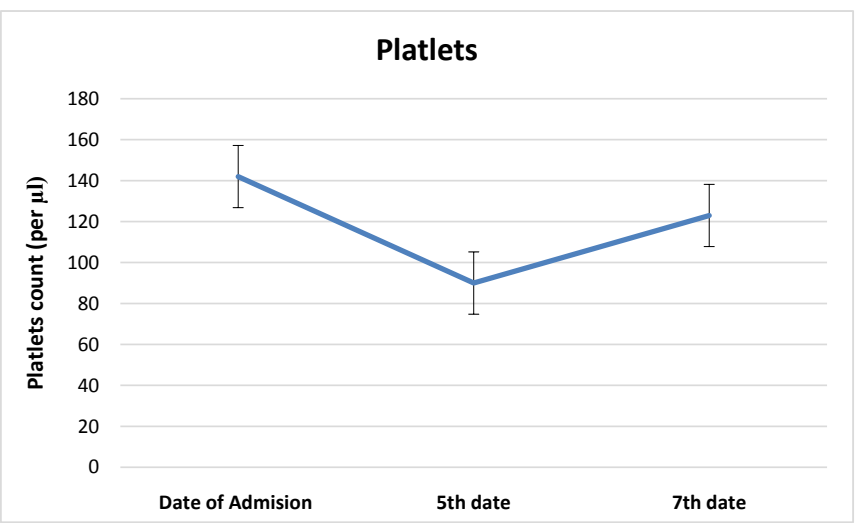

(e)

Figure 1. Changes in serum transaminase levels, antioxidant enzyme and platelets in patients with DF and DHF (a): Changes in serum aspartate transaminase levels; (b): Changes in serum alanine transaminase levels; (c): Changes in serum glutathione reductase; (d): Changes in serum glutathione peroxidase; (e): Changes in platelets.

\section{Discussion}

Fluctuations in antioxidant enzyme levels in the blood and body fluids have been reported for many diseases [23]. Redox disequilibrium is associated with pathology of cardiovascular [20], diabetes and cataracts [19] [21] in our previous studies, where erythrocyte GPX activity was found to be a sensitive marker of 
oxidative stress. Low erythrocyte levels of GPX and GR among patients indicate that superoxides formed by antioxidants of various thiol-containing compounds (glutathione) result in decreased levels of GR. However, in all types of synthesis of GSH, the main source of the tripeptide is produced by the liver.

Therefore, this study was carried out to determine the changes in blood antioxidant enzymes (GR and GPX) in parallel with liver transaminases and other hemaotological parameters in acute phase of dengue viral infection. The results revealed that the concentrations of liver transaminases (AST and ALT) were significantly increased in the day 05 of admission and declined on the day 07 of admission respectively. This trend was more prominent among DHF patients than DF patients. The liver is the main organ which exposes to viral toxicity or immunological injury as response to the viremia. Furthermore, the changes of plasma antioxidants (GR and GPX) levels showed an opposite pattern of release compared to liver enzymes during this acute phase of dengue infection, where the peak reduction of both antioxidants enzyme levels were observed on the day five of admission suggesting that elevation of AST and ALT could be associated with the oxidative stress induced by the dengue virus [5]. The decreased GPX and GR levels reflect an impaired cellular defense mechanism during acute viral phase. Evidence suggests that in the early phase of viral infection perturbation of host cell membrane decreases of intra cellular $\mathrm{pH}$ and GSH efflux which allow to commence the viral cycle in the host cell. Furthermore, it has been documented that GSH also engaged in forming mixed disulfides with viral proteins and consequently GSH levels were further decreased as its component cysteine is incorporated into viral proteins [24]. As a result of decrease GSH concentration which affect the intracellular redox state will which shift more oxidants cause hepatocellular injury and release of liver enzymes (Figure 2), which could require fast reduction of oxidized cysteines allowing replenish the substrate for GSH. Thereby providing $\mathrm{N}$-acetylcystine (NAC), a thiol-containing compound that provides the GSH precursor cysteine, may be more effective in reducing oxidative stress in dengue viral infection [25]. Moreover, it was also reported that oral administration of $\mathrm{N}$-acetyl cysteine restored GSH blood levels in patients with HIV and thereby improved the survival rate [26]. The GPX and GR levels in the study which considered with other diseases such as CVDs, cataract, etc. [19] [20] [21], suggesting that GPX and GR play important role in acute dengue infection. This is thought to be a defense response to increase levels of cellular oxidation and to minimize oxidative damage during pathogenesis of disease.

Although the characteristic primary manifestations in hematological parameters (thrombocytopenia, high hematocrit and leucopenia) in dengue patients were not significant in our study, the changes in hematological parameters were similar to other studies [5]. This could be associated with the damage to cell component, basement membrane and epithelia by lipid peroxidation, protein denaturation and enzyme inactivation in vascular endothelial cells. 


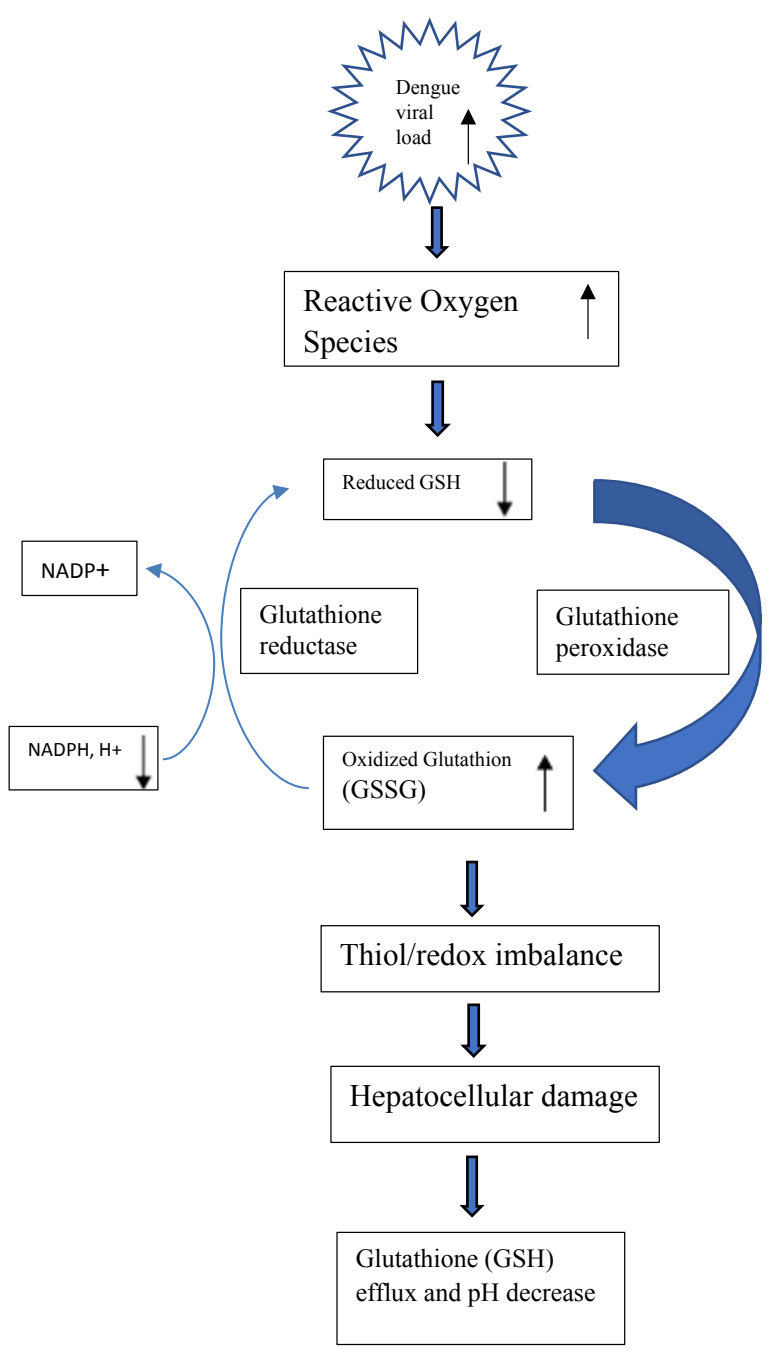

\section{Serum Liver \\ Transaminases}

Figure 2. Changes in intracellular GSH level and hepatocellular damage in dengue viral infection.

In summary, our results indicate that the peak oxidative stress occurs around day five of acute dengue viral infection characterized by elevated hepatocellular enzymes (AST and ALT) and the low antioxidants enzymes (GR and GPX) could be due to high viral load. Thus, timely investigation of antioxidant enzyme levels (GR and GPX) and liver transaminases around day 05 of admission may be of value in the management of patients with dengue infection similar to platelet behavior. This study highlights the importance of glutathione enzymes as a common intermediate for diseases associated with oxidation/redox status such as cataract and CVDs. Furthermore, clinical trials on supplementation of $\mathrm{N}$-acetylcysteine for replenishing the intracellular GSH level will support the contention that NAC supplementation could be useful in acute dengue infection. 


\section{Acknowledgements}

We would like to extend our gratitude to the patients who consented to be part of this study, Ms. D. G. S. Wasana and J. M. Munasinghe and the staff at Nawaloka Chemical Pathology Laboratory for their assistance.

\section{Conflicts of Interest}

The authors declare no conflicts of interest regarding the publication of this paper.

\section{References}

[1] World Health Organization (2009) Dengue: Guidelines for Diagnosis, Treatment, Prevention and Control. Geneva. https://www.who.int/tdr/publications/documents/dengue-diagnosis.pdf

[2] Callaway, E. (2007) Dengue Fever Climbs the Social Ladder. Nature, 448, 734-735. https://doi.org/10.1038/448734a

[3] Weave, S.C. and Vasilakis, N. (2009) Molecular Evolution of Dengue Viruses: Contribution of Phyloge-Netics to Understanding the History and Epidemiology of the Preeminent Arboviral Disease. Infection, Genetics and Evolution, 9, 523-540. https://doi.org/10.1016/j.meegid.2009.02.003

[4] Malavige, G.N., McGowan, S., Atukorale, V., Salimi, M., Peelawatta, M., Fernando, N., Jayaratne, S.D. and Ogg, G. (2012) Identification of Serotype-Specific T Cell Responses to Highly Conserved Regions of the Dengue Viruses. Clinical \& Experimental Immunology, 168, 215-223. https://doi.org/10.1111/j.1365-2249.2012.04566.x

[5] Fernando, S., Wijewickrama, A., Gomes, L., Punchihewa, C.T., Madusanka, S.D., Dissanayake, H., Jeewandara, C., Peiris, H., Ogg, G.S. and Malavige, G.N. (2016) Patterns and Causes of Liver Involvement in Acute Dengue Infection. BMC Infectious Diseases, 16, 319. https://doi.org/10.1186/s12879-016-1656-2

[6] Gubler, D.J. (1998) Dengue and Dengue Haemorrhagic Fever. Clinical Microbiology Review, 11, 480-496.

[7] Malavige, G.N., Fernando, S., Fernando, D.J. and Senevirathne, S. (2004) Dengue Viral Infections. Postgraduate Medical Journal, 80, 588-601. https://doi.org/10.1136/pgmj.2004.019638

[8] Dong, T., Moran, E., Vinh Chau, N., Simmons, C., Luhn, K., Peng, Y., Wills, B., Phuong Dung, N., Thi Thu Thao, L., Hien, T.T., McMichael, A., Farrar, J. and Rowland-Jones, S. (2007) High Pro-Inflammatory Cytokine Secretion and Loss of High Activity Cross-Reactive Cytotoxic T-Cells during the Course of Secondary Dengue Infection. PLoS ONE, 2, c1192. https://doi.org/10.1371/journal.pone.0001192

[9] Mathew, A. and Rothman, A. (2008) Understanding the Contribution of Cellular Immunity to Dengue Disease Pathogenesis. Immunological Reviews, 225, 300-313. https://doi.org/10.1111/j.1600-065X.2008.00678.x

[10] Nkhoma, E.T., Poole, C., Vannappagari, V., Hall, S.A. and Beutler, E. (2009) The Global Prevalence of Glucose-6-Phosphate Dehydrogenase Deficiency: A Systemic Review and Meta-Analysis Systemic Review and Meta-Analysis. Blood Cells, Molecules, and Diseases, 42, 267-278. https://doi.org/10.1016/j.bcmd.2008.12.005

[11] Popovic-Dragonjic, L., Jovanovic, M., Vrbic, M., Konstantinovic, L., Kostic, V. and 
Dragonjic, I. (2011) Antioxidant Defence and Oxidative Stress in Children with Acute Hepatitis A. Annals of Saudi Medicine, 31, 258-262. https://doi.org/10.4103/0256-4947.81538

[12] Wang, Q., Na, B., Ou, J.H., Pulliam, L. and Yen, T.S. (2012) Hepatitis B Virus Alters the Antioxidant System in Transgenic Mice and Sensitizes Hepatocytes to Fas Signaling. PLoS ONE, 7, e36818. https://doi.org/10.1371/journal.pone.0036818

[13] Zwart, L.L, Meerman, J.H.N., Commandeur, J.N.M. and Vermeulen, N.P.E. (1999) Biomarkers of Free Radical Damage. Applications in Experimental Animals and in Humans. Free Radical Biology and Medicine, 26, 202-226. https://doi.org/10.1016/S0891-5849(98)00196-8

[14] Toyokuni, S. (1999) Reactive Oxygen Species-Induced Molecular Damage and Its Application in Pathology. Pathology International, 49, 91-102. https://doi.org/10.1046/j.1440-1827.1999.00829.x

[15] Lizette, G.I.L., Gregorio, M. and Rolando, T. (2004) Oxidative Stress in Adult Dengue Patient. The American Journal of Tropical Medicine and Hygiene, 7, 652-657.

[16] Forman, H.J., Zhang, H. and Rinna, A. (2009) Glutathione: Overview of Its Protective Roles, Measurement, and Biosynthesis. Molecular Aspects of Medicine, 30, 1-12. https://doi.org/10.1016/j.mam.2008.08.006

[17] Kalebic, T., Kinter, A., Poli, G., Anderson, M.E., Meister, A. and Fauci, A.S. (1991) Suppression of Human Immunodeficiency Virus Expression in Chronically Infected Monocytic Cells by Glutathione, Glutathione Ester, and N-Acetylcysteine. Proceedings of the National Academy of Sciences of the United States of America, 88, 986-990. https://doi.org/10.1073/pnas.88.3.986

[18] Dickinson, D.A. and Forman, H.J. (2002) Cellular Glutathione and Thiols Metabolism. Biochemical Pharmacology, 64, 1019-1026. https://doi.org/10.1016/S0006-2952(02)01172-3

[19] Chandrasena, L.G., Chackrewarthy, S., Teckla, P., Perera, M.J. and de Silva, D. (2006) Erythrocyte Antioxidant Enzymes in Patients with Cataract. Annals of Clin ical \& Laboratory Science, 36, 201-204.

[20] Chandrasena, L.G., Peiris, H. and Waikar, H.D. (2009) Biochemical Changes Associated with Reperfusion after off-Pump and on-Pump Coronary Artery Bypass Graft Surgery. Annals of Clinical \& Laboratory Science, 39, 372-376.

[21] Chandrasena, L.G., Peiris, H., Kamani, J., Wanigasuriya, P., Jayaratne, S.D., Wijayasiri, W.A. and Wijesekara, G.U. (2014) Antioxidants in Patients with Dengue Viral Infection. Southeast Asian Journal of Tropical Medicine and Public Health, 45, 1015-1022.

[22] Peterhans, E. (1997) Oxidants and Antioxidants in Viral Disease: Disease Mechanism and Metabolic Regulations. The Journal of Nutrition, 127, 962s-965s. https://doi.org/10.1093/jn/127.5.962S

[23] Canakci, V., Yildirim, A., Canakci, C.F., Eltas, A., Cicek, Y. and Canakci, H. (2007) Total Antioxidant Capacity and Antioxidant Enzymes in Serum, Saliva, and Gingival Crevicular Fluid of Preeclamptic Women with and without Periodontal Disease. Journal of Periodontology, 78, 1602-1611. https://doi.org/10.1902/jop.2007.060469

[24] Nencioni, L., Iuvara, A., Aquilano, K., Ciriolo, M.R., Cozzolino, F., Rotilio, G., Garaci, E. and Palamara, A.T. (2003) Influenza A Virus Replication Is Dependent on an Antioxidant Path-Way that Involves GSH and Bcl-2. The FASEB Journal, 17, 758-760. https://doi.org/10.1096/fj.02-0508fje

[25] Udupi, V. and Rice-Evans, C. (1992) Thiol Compounds as Protective Agents in Erythrocytes under Oxidative Stress. Free Radical Research Communications, 16, 
315-323. https://doi.org/10.3109/10715769209049184

[26] Palamara, A.T., Garaci, E., Rotilio, G., Ciriolo, M.R., Casabianca, A., Fraternale, A., Rossi, L., Schiavano, G.F., Chiarantini, L. and Magnani, M. (1996) Inhibition of Murine AIDS by Reduced Glutathione. AIDS Research and Human Retroviruses, 12, 1373-1381. https://doi.org/10.1089/aid.1996.12.1373 